\title{
Dynamical and geometrical properties of the circular dichroism in one-photon double ionization
}

\author{
J Berakdar $\dagger$ \\ Max-Planck Institute for Microstructure Physics, Weinberg 2, 06120 Halle, Germany
}

Received 2 March 1998

\begin{abstract}
The helicity dependence of the two-electron photoemission from isotropic targets is analysed. As prototype reactions, we consider the double ionization of $\mathrm{H}^{-}\left({ }^{1} \mathrm{~S}^{\mathrm{e}}\right), \mathrm{He}\left({ }^{1} \mathrm{~S}^{\mathrm{e}}\right)$, $\mathrm{Li}^{+}\left({ }^{1} \mathrm{~S}^{\mathrm{e}}\right), \mathrm{Be}^{2+}\left({ }^{1} \mathrm{~S}^{\mathrm{e}}\right)$ and $\mathrm{B}^{3+}\left({ }^{1} \mathrm{~S}^{\mathrm{e}}\right)$ upon the absorption of a circularly polarized photon. Assuming the Coulomb field of the nucleus to be dominant over other involved interactions, we derive analytical expressions for the cross sections and their dependence on the photon helicity, i.e. for the circular dichroism in these cross sections. This analysis indicates that the cross sections and the circular dichroism are influenced in a markedly different manner by the interelectronic correlation. With increasing strength of the Coulomb nuclear field the cross section rapidly diminishes whilst the dichroism remains finite and its sign varies considerably with the nuclear charge.

It is analytically concluded that the absence of the dichroism at certain 'non-geometrical' points as well as at certain intermediate excess energies is a direct manifestation of the interelectronic correlation. At threshold and in the high-energy limit negligible dichroism is anticipated.
\end{abstract}

\section{Introduction}

It is established (Berakdar and Klar 1992, Berakdar et al 1993, Kabachnik and Schmidt 1995, Manakov et al 1996, Viefhaus et al 1996, Mergel et al 1998) that the two-electron ejection from a randomly oriented target following the absorption of circularly polarized light is strongly dependent on the helicity of the photon. In contrast to the case of single photoionization, this circular dichroism (CD) persists even if the magnetic substates of the photoion and/or the spin states of the photoelectrons are not resolved.

Using a tensorial recoupling scheme and a formal partial-wave expansion of the continuum wavefunction of the two electrons in the field of the photoion, the existence of the $\mathrm{CD}$ has been documented. In addition, a vanishing $\mathrm{CD}$ has been predicted at geometrical arrangements for which the vectors $\boldsymbol{k}_{a}, \boldsymbol{k}_{b}, \boldsymbol{k}$ are linearly dependent or $\left|\boldsymbol{k}_{a}\right|=\left|\boldsymbol{k}_{b}\right|$ (these conditions are called hereafter 'geometrical zero points' of the CD), where $\boldsymbol{k}_{a}, \boldsymbol{k}_{b}$ are the vector momenta of the escaping electrons and $\hat{\boldsymbol{k}}$ is the wavevector of the photon. These studies are the formal fundament for the existence of the circular dichroism, yet they leave in the dark the actual magnitude, sign and behaviour of the CD.

Predictions of dynamical calculations using approximate two-electron wavefunctions (Berakdar and Klar 1992, Berakdar et al 1993) underlined the existence of an observable CD as well as the geometrical zero points. In addition, these calculations revealed a complicated

$\dagger$ E-mail address: jber@mpi-halle.de 
behaviour of the CD that could not be interpreted within the formal analysis: at certain, $a$ priori not clear, angular and energy positions of the outgoing electrons the $\mathrm{CD}$ vanishes and changes sign. Furthermore, a strong dependence of the $\mathrm{CD}$ on the photon frequency has been observed. The origin of this behaviour has not yet been clarified.

Currently there is a considerable interest in the experimental investigation of this kind of dichroic effects (Soejima et al 1996, Viefhaus et al 1996, Mergel et al 1998) as a new feature of many-body correlated systems. On the other hand, the important and exciting applications of $\mathrm{CD}$ in single photoemission for determining structural and (macroscopic) magnetic properties of thin films (Venus et al 1997) fuelled the hope that the present CD can provide an insight in the electronic correlation of such systems. Thus it is appropriate to analyse in detail the influence of the individual interactions on the $\mathrm{CD}$ and to clarify the role of electronic correlation and its manifestation in the CD. In addition, there is as yet no analysis of the element-dependent aspects of the $\mathrm{CD}$. Therefore, the $\mathrm{CD}$ is investigated for a variety of targets.

We study at first the interrelation between the $\mathrm{CD}$ and the strength of the Coulomb nuclear field by switching off the interelectronic correlation. Without any further approximations, analytical expressions are derived for the $\mathrm{CD}$ and the corresponding cross sections. From these formulae the following conclusions are made.

(1) Within a model of spatially decoupled electrons, the CD as a function of $\hat{\boldsymbol{k}}_{a}, \hat{\boldsymbol{k}}_{b}$, and $\boldsymbol{k}$ does not vanish except for the 'geometrical points'.

(2) The $\mathrm{CD}$ as a function of the photon frequency only diminishes at the doubleionization threshold and in the high-energy limit.

(3) With increasing strength of the Coulomb nuclear field the dichroism remains finite whilst the cross section rapidly decreases.

Now combining these findings with calculations that (approximately) account for electronic correlation we conclude that a vanishing $\mathrm{CD}$ at the non-geometrical points as well as at some intermediate photon frequencies is solely due to electronic coupling.

It is reasonable to presume that this coupling plays a subsidiary role for highly charged residual ions. Consequently, in this case one can expect that the analytical formulae derived here are useful.

The derived results are tested in the cases of the double ionization of $\mathrm{H}^{-}\left({ }^{1} \mathrm{~S}^{\mathrm{e}}\right), \mathrm{He}\left({ }^{1} \mathrm{~S}^{\mathrm{e}}\right)$, $\mathrm{Li}^{+}\left({ }^{1} \mathrm{~S}^{\mathrm{e}}\right), \mathrm{Be}^{2+}\left({ }^{1} \mathrm{~S}^{\mathrm{e}}\right)$ and $\mathrm{B}^{3+}\left({ }^{1} \mathrm{~S}^{\mathrm{e}}\right)$ following the absorption of a circularly polarized photon. The first of these targets is a prototype of strong interelectronic coupling whereas for the last one the interaction with the nucleus is regarded as the 'strong potential'. Here we focus on the geometry of the first experiment in which the CD has recently been observed (Viefhaus et al 1996).

Throughout this paper atomic units are employed and the $\boldsymbol{L} \cdot \boldsymbol{S}$ interaction is neglected.

\section{Theoretical models}

The double ionization with circularly polarized light can be quantified by the independent functions

$$
\mathrm{CD}:=\frac{\operatorname{TDCS}\left(\sigma^{+}\right)-\operatorname{TDCS}\left(\sigma^{-}\right)}{\operatorname{TDCS}\left(\sigma^{+}\right)+\operatorname{TDCS}\left(\sigma^{-}\right)}
$$

and, optionally, $\operatorname{TDCS}\left(\sigma^{+}\right)$, where $\operatorname{TDCS}\left(\sigma^{+}\right)\left(\operatorname{TDCS}\left(\sigma^{-}\right)\right)$is the triply (fully) differential cross section for double ionization by left (right) circularly polarized photon; in the notation of Jackson (1967) this corresponds to positive (negative) photon helicity. 
Hence, the dynamical and geometrical properties of the CD and $\operatorname{TDCS}\left(\sigma^{+}\right)$, in particular their dependence on the radial and angular interelectronic correlation, are not related to each other.

As the CD and the $\operatorname{TDCS}\left(\sigma^{+}\right)$are independent facets of the (correlated) electron-pair emission upon single-photon absorption, an observable CD can be regarded as footprints for this simultaneous emission in situations where measuring the $\operatorname{TDCS}\left(\sigma^{ \pm}\right)$does not provide unique information as to whether the escaping electrons are emitted due to the direct absorption of the photon or other secondary reactions following single photoemission (the $\mathrm{CD}$ vanishes in this case). The latter process is expected to be of prominent importance in the one-photon two-electron emission from condensed matter (Herrmann et al 1998, Berakdar 1998).

The present analysis is based on the first-order perturbation theory and on the dipole approximation for the radiation field. The length form is used throughout.

Upon the absorption of a photon of frequency $\omega$, the TDCS for the emission of two electrons under the solid angles of aperture $\mathrm{d} \Omega_{a}$ and $\mathrm{d} \Omega_{b}$, one of them having energy $E_{b}$, is

$$
\operatorname{TDCS}:=\frac{\mathrm{d}^{3} \sigma}{\mathrm{d} \Omega_{a} \mathrm{~d} \Omega_{b} \mathrm{~d} E_{b}}=C \sum_{M_{f}} \frac{1}{2 J_{i}+1} \sum_{M_{i}}\left|\left\langle\Psi_{\boldsymbol{k}_{a}, \boldsymbol{k}_{b}}^{-}|\hat{\boldsymbol{\epsilon}} \cdot D| \Phi_{i}\right\rangle\right|^{2},
$$

where $\hat{\boldsymbol{\epsilon}}$ is the complex polarization vector of the photon, $C=4 \pi^{2} \alpha_{c} k_{a} k_{b} \omega, E_{b}=k_{b}^{2} / 2$, and $\alpha_{c}$ is the fine-structure constant. Equation (2) averages over the initial magnetic sublevels $M_{i}$, and sums over the magnetic sublevels $M_{f}$ of the photoion. This study is confined to two-electron targets with ${ }^{1} \mathrm{~S}$ symmetry. The dipole operator $\boldsymbol{D}$ is then $\boldsymbol{D}=\boldsymbol{r}_{a}+\boldsymbol{r}_{b}$ where $\boldsymbol{r}_{a / b}$ are the positions of the two electrons with respect to the nucleus. The initial and the final states are, respectively, described by the three-body wavefunctions $\Phi_{i}\left(\boldsymbol{r}_{a}, \boldsymbol{r}_{b}\right)$ and $\Psi_{\boldsymbol{k}_{a}, \boldsymbol{k}_{b}}^{-}\left(\boldsymbol{r}_{a}, \boldsymbol{r}_{b}\right)$, which have to be approximated in order to get some estimate of the cross section (2). To systematically investigate the influence of various interactions on the CD and the $\operatorname{TDCS}\left(\sigma^{ \pm}\right)$we employ deliberately simple expressions for the ${ }^{1} \mathrm{~S}^{\mathrm{e}}$ initial state:

$$
\begin{aligned}
& \Phi_{s}=N_{s} \exp \left[-Z_{s}\left(r_{a}+r_{b}\right)\right] \\
& \Phi_{3 h}=N_{h}\left[\exp \left(-\alpha_{h 1} r_{a}-\alpha_{h 2} r_{b}\right)+\exp \left(-\alpha_{h 1} r_{b}-\alpha_{h 2} r_{a}\right)\right] \exp \left(\beta r_{a b}\right) .
\end{aligned}
$$

The parameters $Z_{s}, \alpha_{h 1 / h 2}, \beta$ are variationally determined by minimizing the binding energy and $N_{s / h}$ are normalization factors. A Ritz variational procedure (Bethe and Salpeter 1957) yields for (3): $Z_{s}=Z-\frac{5}{16}, N_{s}=Z_{s}^{3} / \pi$, where $Z$ is the nuclear charge. This means by using (3) we account for the electron-electron interaction as a merely effective (angular and radially independent) screening of the nuclear interaction. According to (3), in the limit of $Z \gg 1$ we obtain $Z_{s} \approx Z$, i.e. the electron-electron interaction can be neglected altogether in favour of the nuclear one.

Representing the initial state by equation (4) we (approximately) account for radial and angular interelectronic coupling.

The final-state wavefunction is modelled by the symmetrized form

$$
\Psi_{\boldsymbol{k}_{a}, \boldsymbol{k}_{b}}^{S}\left(\boldsymbol{r}_{a}, \boldsymbol{r}_{b}\right)=\left[\Psi_{\boldsymbol{k}_{a}, \boldsymbol{k}_{b}}^{-}\left(\boldsymbol{r}_{a}, \boldsymbol{r}_{b}\right)+\Psi_{\boldsymbol{k}_{a}, \boldsymbol{k}_{b}}^{-}\left(\boldsymbol{r}_{b}, \boldsymbol{r}_{a}\right)\right] / \sqrt{2}
$$

The factor $1 / \sqrt{2}$ follows from the requirement that $\Psi^{S}$ has to be normalized to delta functions in the six-dimensional space spanned by $\boldsymbol{k}_{a} \otimes \boldsymbol{k}_{b}$ (the conjugate space to $\boldsymbol{r}_{a} \otimes \boldsymbol{r}_{b}$ ), i.e. $\left\langle\Psi_{\boldsymbol{k}_{a}, \boldsymbol{k}_{b}}^{S}\left(\boldsymbol{r}_{a}, \boldsymbol{r}_{b}\right) \mid \Psi_{\boldsymbol{k}_{a}^{\prime}, \boldsymbol{k}_{b}^{\prime}}^{S}\left(\boldsymbol{r}_{a}, \boldsymbol{r}_{b}\right)\right\rangle=\delta\left(\boldsymbol{k}_{a}^{\prime}-\boldsymbol{k}_{a}\right) \delta\left(\boldsymbol{k}_{b}^{\prime}-\boldsymbol{k}_{b}\right)$. The functions $\Psi_{\boldsymbol{k}_{a}, \boldsymbol{k}_{b}}^{-}\left(\boldsymbol{r}_{a}, \boldsymbol{r}_{b}\right)$ are already normalized in this way. Thus in order to prove that $\Psi_{k_{a}, \boldsymbol{k}_{b}}^{S}\left(\boldsymbol{r}_{a}, \boldsymbol{r}_{b}\right)$ (equation (5)) is correctly normalized it suffices to show that

$$
\left\langle\Psi_{\boldsymbol{p}_{a}, \boldsymbol{p}_{b}}^{-}\left(\boldsymbol{r}_{a}, \boldsymbol{r}_{b}\right) \mid \Psi_{\boldsymbol{p}_{a}^{\prime}, \boldsymbol{p}_{b}^{\prime}}^{-}\left(\boldsymbol{r}_{b}, \boldsymbol{r}_{a}\right)\right\rangle=0, \quad \forall \boldsymbol{p}_{a}, \boldsymbol{p}_{a}^{\prime} ; \boldsymbol{p}_{b}, \boldsymbol{p}_{b}^{\prime} \in \boldsymbol{k}_{a} \otimes \boldsymbol{k}_{b}
$$


To prove (6) we note that the total Hamiltonian is invariant under the exchange of labelling $a \longleftrightarrow b$. This imposes on the eigenstates of this Hamiltonian the symmetry requirement $\Psi_{\boldsymbol{p}_{a}, \boldsymbol{p}_{b}}^{-}\left(\boldsymbol{r}_{a}, \boldsymbol{r}_{b}\right)=\Psi_{\boldsymbol{p}_{b}, \boldsymbol{p}_{a}}^{-}\left(\boldsymbol{r}_{b}, \boldsymbol{r}_{a}\right)$. Equation (6) can thus be rewritten as

$$
\left\langle\Psi_{\boldsymbol{p}_{a}, \boldsymbol{p}_{b}}^{-}\left(\boldsymbol{r}_{a}, \boldsymbol{r}_{b}\right) \mid \Psi_{\boldsymbol{p}_{b}^{\prime}, \boldsymbol{p}_{a}^{\prime}}^{-}\left(\boldsymbol{r}_{a}, \boldsymbol{r}_{b}\right)\right\rangle=\delta\left(\boldsymbol{p}_{b}^{\prime}-\boldsymbol{p}_{a}\right) \delta\left(\boldsymbol{p}_{a}^{\prime}-\boldsymbol{p}_{b}\right)=0 .
$$

Equation (7) follows from the fact that $\boldsymbol{p}_{a}$ and $\boldsymbol{p}_{b}^{\prime}$ (as well as $\boldsymbol{p}_{b}$ and $\boldsymbol{p}_{a}^{\prime}$ ) signify independent subspaces of $\boldsymbol{k}_{a} \otimes \boldsymbol{k}_{b}$ (in fact $\boldsymbol{k}_{a} \otimes \boldsymbol{k}_{b}$ is spanned by the set $\left|\boldsymbol{p}_{a}\right\rangle \otimes\left|\boldsymbol{p}_{b}^{\prime}\right\rangle$ ).

Thus, a normalized symmetric expression, such as equation (5), can always be constructed from wavefunctions that are invariant under the operation $a \longleftrightarrow b$. As wavefunctions that possess this symmetry property we employ

$$
\begin{aligned}
& \Psi_{\boldsymbol{k}_{a}, \boldsymbol{k}_{b}}^{-}\left(\boldsymbol{r}_{a}, \boldsymbol{r}_{b}\right) \approx \Psi_{3 C}:=(2 \pi)^{-3} N_{a} N_{b} N_{a b} \mathrm{e}^{\mathrm{i} \boldsymbol{k}_{a} \cdot \boldsymbol{r}_{a}+\mathrm{i} \boldsymbol{k}_{b} \cdot \boldsymbol{r}_{b}}{ }_{1} F_{1}\left[\mathrm{i} \beta_{a}, 1,-\mathrm{i}\left(k_{a} r_{a}+\boldsymbol{k}_{a} \cdot \boldsymbol{r}_{a}\right)\right] \\
& \times{ }_{1} F_{1}\left[\mathrm{i} \beta_{b}, 1,-\mathrm{i}\left(k_{b} r_{b}+\boldsymbol{k}_{b} \cdot \boldsymbol{r}_{b}\right)\right]_{1} F_{1}\left[\mathrm{i} \beta_{a b}, 1,-\mathrm{i}\left(k_{a b} r_{a b}+\boldsymbol{k}_{a b} \cdot \boldsymbol{r}_{a b}\right)\right], \\
& \Psi_{2 C}=\left.\Psi_{3 C}\right|_{\beta_{a b} \equiv 0},
\end{aligned}
$$

and

$$
\Psi_{2 C N}=N_{a b} \Psi_{2 C} \text {. }
$$

In equation (8) we defined $\boldsymbol{r}_{a b}:=\boldsymbol{r}_{a}-\boldsymbol{r}_{b}$ and $\boldsymbol{k}_{a b}$ as its conjugate momentum. The Sommerfeld parameters are given by $\beta_{a / b}:=-Z / k_{a / b}, \beta_{a b}:=1 / 2 k_{a b}$, and the normalization constants by $N_{j}=\exp \left(-\pi \beta_{j} / 2\right) \Gamma\left(1-\mathrm{i} \beta_{j}\right), j=a, b, a b \dagger$. Properties of the wavefunction (8) have been discussed elsewhere (Brauner et al 1989). In the context of this study it is important to note that the approximations made to arrive at equation (9) regard the two escaping electrons as independent particles moving in the field of the nucleus. In equation (10) the electron-electron repulsion enters via the two-electron Coulomb density of states which is proportional to $\left|N_{a b}\right|^{2}$. No radial or angular coupling between the two electrons is included in equation (10). In equation (8) all two-body interactions are treated on equal footing. With regard to this work we note that the expressions (8), (10) turned out to be quite adequate for the description of the angular distribution of two emitted electrons from $\operatorname{He}\left({ }^{1} \mathrm{~S}^{\mathrm{e}}\right)$ upon the absorption of a linearly polarized photon (Maulbetsch et al 1995, Maulbetsch 1995). However, as the polarization of the photon enters dynamically into the cross section, it is not clear how these approximations will perform in the present case of circular polarization. Furthermore, the approximations (3), (9) become more reasonable for higher nuclear charge $Z \gg 1$. In fact, equation (3) predicts ground-state binding energies that are in increasingly better agreement with experimental findings at higher $Z$. In contrast, the correlated wavefunction (8) is not expected to perform well for $Z \gg 1$ as it does not contain any screening of the electronic interaction due to the presence of the nuclear charge (Berakdar 1996).

\section{Geometry and dynamics of the CD}

Since the target is randomly oriented, the relative dichroism

$$
\Delta:=\operatorname{TDCS}\left(\sigma^{+}\right)-\operatorname{TDCS}\left(\sigma^{-}\right)
$$

must be, like all observables, invariant under exchange of the two electrons, i.e.

$$
\Delta\left(\boldsymbol{k}_{a}, \boldsymbol{k}_{b}\right)=\Delta\left(\boldsymbol{k}_{b}, \boldsymbol{k}_{a}\right) .
$$

$\dagger$ The analysis is easily repeated for velocity-dependent effective Sommerfeld parameters with basically the same conclusions as drawn here. 
Using the expressions (10), (3), $\Delta\left(\boldsymbol{k}_{a}, \boldsymbol{k}_{b}\right)$ can be evaluated in closed form (see appendix A):

$$
\Delta=-Z F\left(k_{a}-k_{b}\right)\left(\hat{\boldsymbol{k}}_{a} \times \hat{\boldsymbol{k}}_{b}\right) \cdot \hat{\boldsymbol{k}} .
$$

The function $F$ reads

$$
F=2 C_{a}\left(Z_{s}-Z\right)^{2}\left(2 Z_{s}-Z\right)^{2}\left(2 f_{a} f_{b}\right)^{2}\left(k_{a}^{2}+Z_{s}^{2}\right)^{-5}\left(k_{b}^{2}+Z_{s}^{2}\right)^{-5},
$$

where $C_{a}$ and $f_{j}, j=a, b$ are given by equation (A3) and equation (A6), respectively. The geometrical properties of $\Delta$, in particular the zero points of $\Delta$ that are described in the introduction, are entirely expressed in the triple vectorial product in equation (12) and the factor $\left(k_{a}-k_{b}\right) . \quad F$ contains the dynamical information. Since $\left(k_{a}-k_{b}\right)$ and $\left(\hat{\boldsymbol{k}}_{a} \times \hat{\boldsymbol{k}}_{b}\right) \cdot \hat{\boldsymbol{k}}$ are both antisymmetric with respect to exchange of the two electrons, we deduce $F\left(k_{a}, k_{b}\right)=F\left(k_{b}, k_{a}\right)$, for equation (11) must apply. Furthermore, upon inspection of $F\left(k_{a}, k_{b}\right.$ ) (equation (13)) we verify that $F$ is positive definite for all combinations of $\boldsymbol{k}_{a}$ and $\boldsymbol{k}_{b}$. This means that, within the approximations (3), (9), $\Delta$ does not vanish except for the points dictated by the geometrical part. This conclusion is also valid for the normalized dichroism CD: $=\Delta / \Sigma$ (see equation (B1)), where $\Sigma:=\operatorname{TDCS}\left(\sigma^{+}\right)+\operatorname{TDCS}\left(\sigma^{-}\right)$, since $F / \Sigma$ is also positive definite $(\Sigma>0)$. In addition, the final-state wavefunctions (10) and (9) yield the same CD because the factor $\left|N_{a b}\right|^{2}$ does not depend on the polarization vector and does not enter the matrix element in a dynamical way. In contrast, the predictions for the TDCS using equations (10) and (9) are markedly different, as demonstrated by Maulbetsch et al (1995) and Maulbetsch (1995) (see also below). These observations apply for all wavefunctions that contain the interelectronic interaction via a coordinate-independent multiplicative factor.

At this stage we summarize, that any zero points in the CD other than the geometrical ones (described by $\left.\left(k_{a}-k_{b}\right)\left(\hat{\boldsymbol{k}}_{a} \times \hat{\boldsymbol{k}}_{b}\right) \cdot \hat{\boldsymbol{k}}\right)$ are attributed to electron-electron coupling that goes beyond equation (10).

\subsection{Numerical examples}

To illustrate the preceding arguments we consider the geometry under which the CD has recently been measured (Viefhaus et al 1996) (for $\mathrm{He}\left({ }^{1} \mathrm{~S}^{\mathrm{e}}\right)$ ). The wavevector of the photon $\hat{\boldsymbol{k}}$ was chosen perpendicular to the plane spanned by the vectors $\boldsymbol{k}_{a}$ and $\boldsymbol{k}_{b}$. For a fixed interelectronic angle $\theta_{a b}:=\cos ^{-1} \hat{\boldsymbol{k}}_{a} \cdot \hat{\boldsymbol{k}}_{b}\left(\theta_{a b}=150^{\circ}, 125^{\circ}, 85^{\circ}\right)$ the CD and the TDCS were measured as a function of the excess-energy sharing between the two electrons. For a given $\theta_{a b}$ the relative magnitude of the TDCS with respect to the other measurements at different $\theta_{a b}$ has been determined by the experiment. The absolute value of the TDCS at a specific measured point is, however, unknown. The $\operatorname{TDCS}\left(\sigma^{+}\right)$maps onto $\operatorname{TDCS}\left(\sigma^{-}\right)$via a reflection at the line $E_{b}=E / 2$, where $E$ is the (constant) excess energy. In figure 1 these data are depicted along with the predictions of the model employing (3), (9), the analytical formulae are derived in the appendices A and B. It is clear that using the uncorrelated wavefunctions (3), (9) leads to theoretical predictions for the TDCS which are not in accord with the experimental finding. The $\mathrm{CD}$, however, is reasonably well described by these electronically uncorrelated models. If we allow for the final-state interelectronic correlation (figure 2) by simply using equation (10) we leave the CD unchanged, as argued above, yet we clearly improve on describing the TDCS data. The change in the TDCS (shown in figure 1) in response to using the wavefunction (10) (figure 2) is readily understood by analysing the factor $\left|N_{a b}\right|^{2}$ (see figure 3). When the two electrons escape in opposite directions the electron-electron repulsion is minimized for equal-energy sharing, i.e. $\left|N_{a b}\right|^{2}$ is largest in 

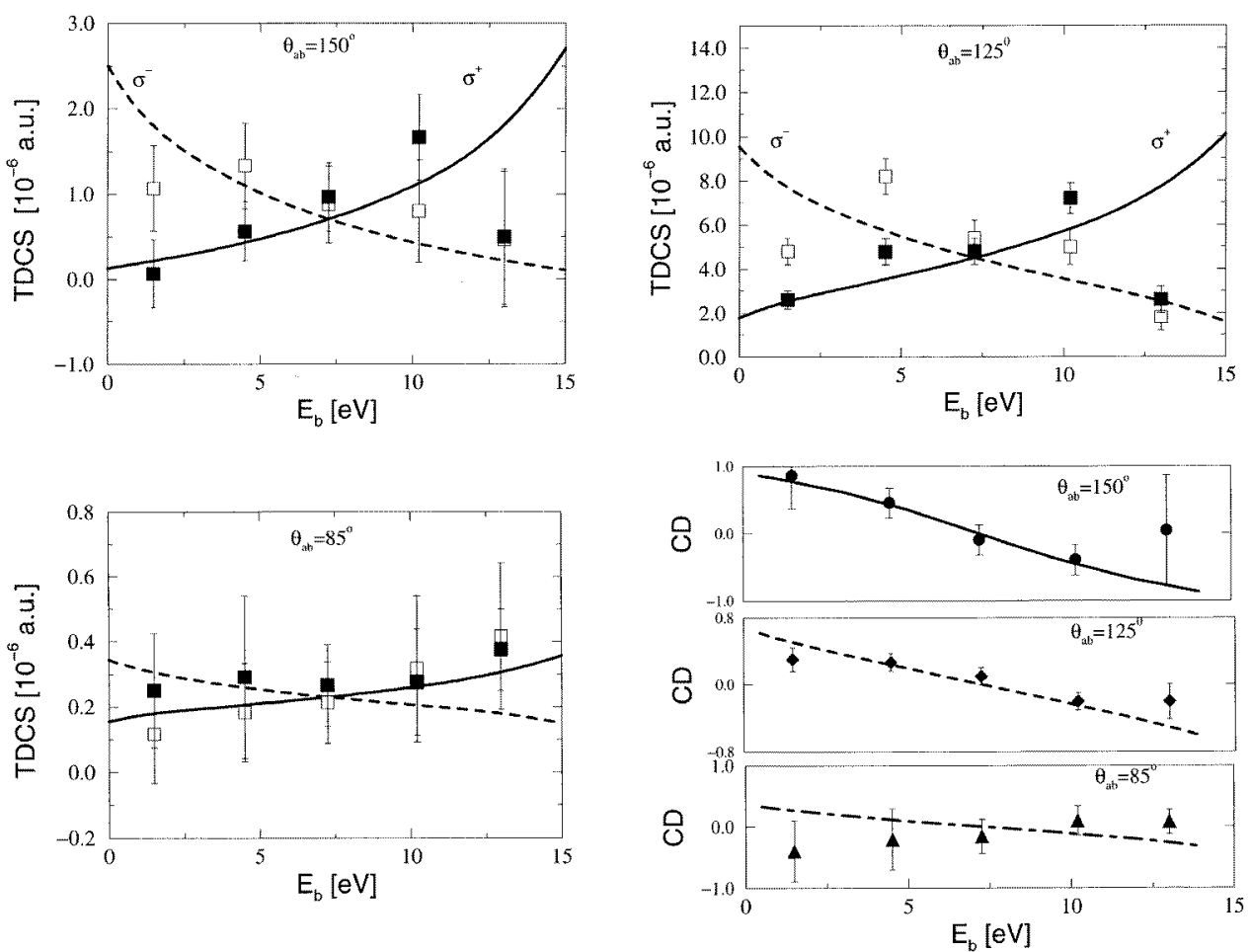

Figure 1. The TDCS $\left(\sigma^{+}\right)$(full curve) and $\operatorname{TDCS}\left(\sigma^{-}\right)$(broken curve) for the one-photon double ionization of $\operatorname{He}\left({ }^{1} \mathrm{~S}^{\mathrm{e}}\right)$. The theory employs the wavefunctions (3), (9) for the initial and final states, respectively. The wavevector $\hat{k}$ of the photon is chosen as the $z$-direction. The two electrons are detected in the upper half of the $x y$-plane with electron 'a' fixed along the $x$-axis and electron 'b' detected under an angle $\theta_{a b}$ with respect to the first one. The angles $\theta_{a b}$ are indicated in the figures. The excess energy is $14.52 \mathrm{eV}$. The experimental data (full squares for $\sigma^{+}$and open squares for $\sigma^{-}$) are due to Viefhaus et al (1996). The theoretical results for $\theta_{a b}=150^{\circ}\left(85^{\circ}\right)$ have been scaled down by a factor of $2(50)$. In addition the corresponding normalized $\mathrm{CD}$ is separately depicted.

this case. In contrast, for forward emission of the two electrons the interelectronic coupling is weakest for asymmetric-energy sharing. Thus, when including the electron-electron repulsion, in the way it is done in equation (10), the TDCS shown in figure 1 bends at asymmetric energies for $\theta_{a b}=150^{\circ}, 125^{\circ}$, but remains almost unaffected for $\theta_{a b}=85^{\circ}$.

The experimental data indicate an inverted sign behaviour of the $\mathrm{CD}$ at $\theta_{a b}=85^{\circ}$, i.e. at small $E_{b}$ the $\mathrm{CD}$ goes from positive to negative by varying $\theta_{a b}$ from $150^{\circ}$ to $85^{\circ}$. This means that the $\mathrm{CD}$ vanishes at a certain angle in the interval $\theta_{a b} \in\left[85^{\circ}, 150^{\circ}\right]$. Owing to the transformation properties of $\operatorname{TDCS}\left(\sigma^{ \pm}\right)$it follows that at this point $\operatorname{TDCS}\left(\sigma^{-}\right) \equiv$ $\operatorname{TDCS}\left(\sigma^{+}\right)$possesses symmetric energy sharing with respect to $k_{a}=k_{b}$. As stated above, such a change of sign is prohibited within the 'uncorrelated' models which employ equations (3), (9) and (10). This does not apply for the wavefunction (8) that includes, in addition to $N_{a b}$, the radial part of the electron-electron Coulomb wave. Unfortunately, it has not yet been possible to obtain the $\mathrm{CD}$ in closed form for this case. The numerical results are shown in figure 4 that clearly show the changed sign as indicated by the experiment (cf Viefhaus et al 1996). This confirms the conclusion of the preceding section in that a sign change of the $\mathrm{CD}$ is a footprint of spatial interelectronic coupling. 

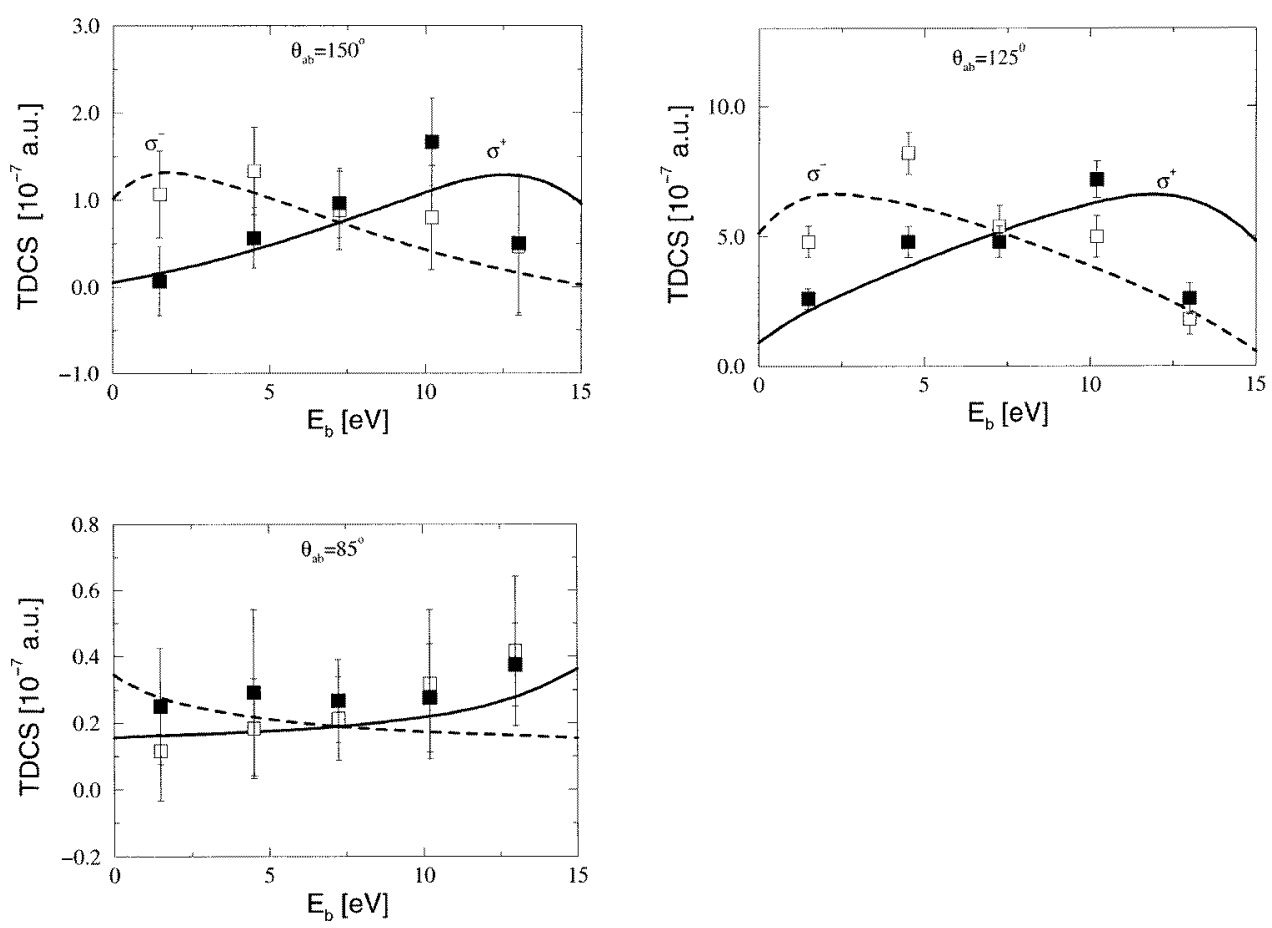

Figure 2. The same as in figure 1, however, the final-state wavefunction (10) has been used. The theory for $\theta_{a b}=150^{\circ}\left(85^{\circ}\right)$ has been scaled down by a factor of $3(20)$.

As mentioned earlier, the experimental data are internormalized. the models employing equations (9), (10) fail to reproduce the measured internormalization factor. Using the correlated final state (8) improves on the agreement with experiment, yet it does not completely resolve these discrepancies. It turned out (Berakdar 1997 (results not included here for brevity)) that these shortcoming can be satisfactorily circumvented by using the highly correlated initial-state wavefunction of Siebbeles et al (1993) and equation (8) for the final state.

A further important point is the absolute size of the $\mathrm{CD}$. The general trend is observed in figure 1. From first sight it appears surprising that the $C D$ decreases substantially when we decrease the angle $\theta_{a b}$ from $150^{\circ}$ to $85^{\circ}$, as the geometrical factor in equation (12) indicates a maximum $\mathrm{CD}$ for $\theta_{a b}=90^{\circ}$. To understand this behaviour we analyse the analytical formula for the $\mathrm{CD}$,

$$
\mathrm{CD}=-\frac{Z}{\mathcal{F}}\left(k_{a}-k_{b}\right)\left(\hat{\boldsymbol{k}}_{a} \times \hat{\boldsymbol{k}}_{b}\right) \cdot \hat{\boldsymbol{k}}
$$

where $\mathcal{F}=\Sigma / F$ is given by equation (B6). As $\Sigma$ and $F$ are positive definite functions of $k_{a}$ and $k_{b}$, we conclude $\mathcal{F}\left(k_{a}, k_{b}\right)>0$. For the present geometry $\mathcal{F}$ simplifies to

$$
\mathcal{F}=\frac{k_{b}^{2}+Z_{s}^{2}}{k_{a}^{2}+Z_{s}^{2}}\left(k_{a}^{2}+Z^{2}\right)+\frac{k_{a}^{2}+Z_{s}^{2}}{k_{b}^{2}+Z_{s}^{2}}\left(k_{b}^{2}+Z^{2}\right)+2\left(k_{a} k_{b}+Z^{2}\right) \cos \theta_{a b} .
$$

From equation (15) it is obvious that $\mathcal{F}$ is largest (smallest) for forward (backward) emission of both electrons, i.e. $\theta_{a b} \approx 0\left(\theta_{a b} \approx \pi\right)$. This dependence is directly reflected into the $\mathrm{CD}$, according to equation (14), and leads to the behaviour observed in figures 1 and 4 (note, however, that the $\mathrm{CD}$ vanishes at $\theta_{a b}=0, \pi$ due to the geometrical factors in equation (14)). 


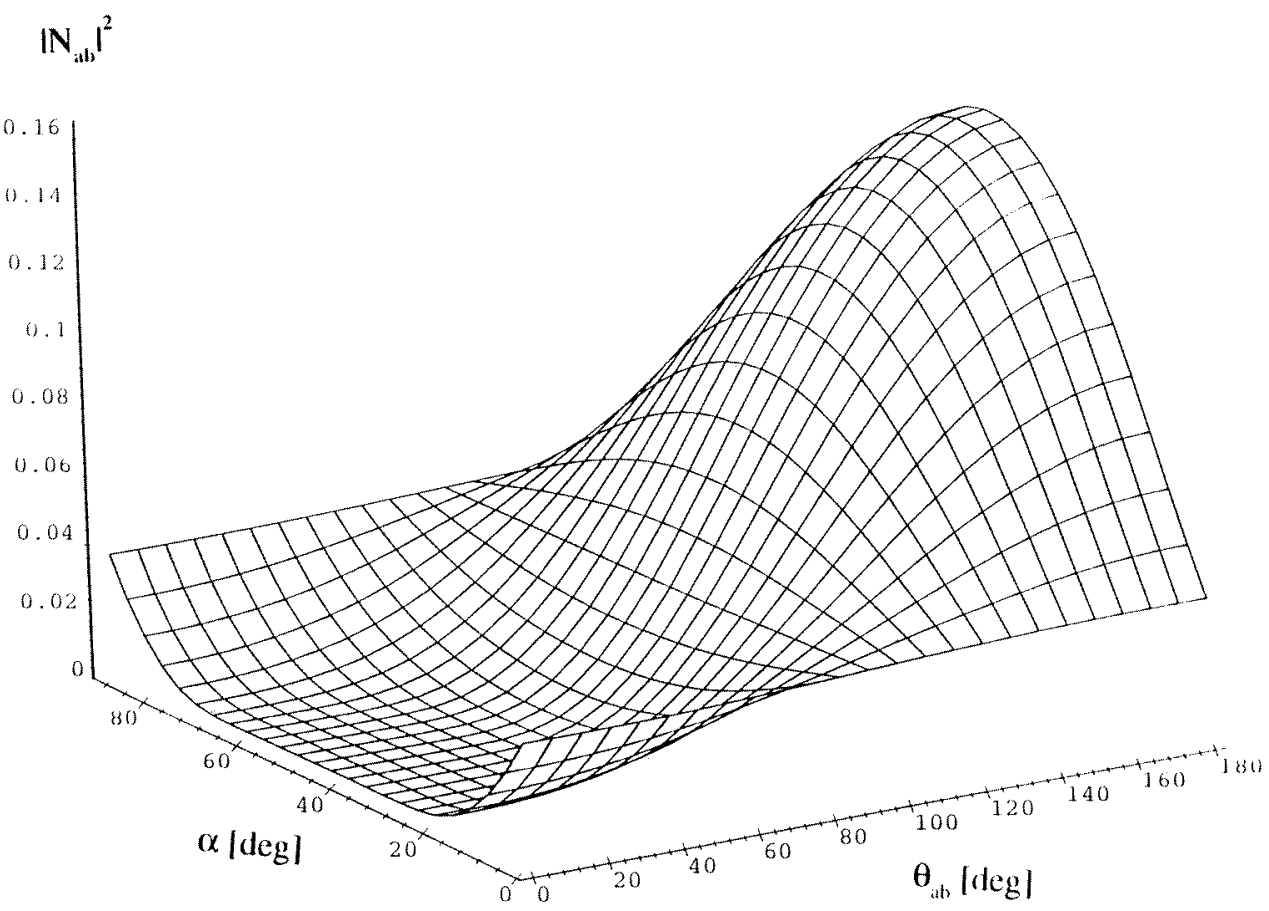

Figure 3. The factor $\left|N_{a b}\right|^{2}$ that enters into equation (10) plotted, at an excess energy of $14.52 \mathrm{eV}$, as a function of $\alpha:=\tan ^{-1} k_{a} / k_{b}$ and $\theta_{a b}$.

\section{The dependence of the CD and $\Sigma$ on the nuclear charge}

As previously noted, the approximate forms (9), (3) become more appropriate with increasing nuclear charge $Z$. Hence, it is worthwhile to study the CD and the TDCS for moderately large $Z$ (for $Z \gg 1$ the present formalism is not valid, for it neglects the $\boldsymbol{L} \cdot \boldsymbol{S}$ interaction). For higher $Z$ the screening of the nuclear charge due to the electronic interaction becomes more and more insignificant, i.e. $\lim _{Z \gg 1} Z_{s} \rightarrow Z$. In this case the double photoionization becomes more unlikely, as readily deduced from equation (B4) (for $Z_{s}=Z$ equations (3) and (9) are then uncorrelated solutions of the same Hamiltonian for different eigenvalues, consequently, the TDCS, given by equation (2), vanishes). In contrast, the CD (see equations (14), (15)) remains, in general, finite.

The dependence of the $\mathrm{CD}$ on $Z$ is linked to the geometry of the experiment. For example, if we consider the $\mathrm{CD}$ as a function of $\theta_{a b}$ for energies of the electrons such that $k_{a} \gg Z \ll k_{b}$, then the $Z$-dependence of $\mathcal{F}$ can be neglected and the CD increases with increasing $Z$. For very asymmetric energy sharing, however, the function $\mathcal{F}$ (equation (15)) becomes proportional to $Z^{2}$ for large nuclear charge. Therefore the $\mathrm{CD}$ decreases with increasing $Z$. The latter behaviour can be seen in figure 5 for $\mathrm{Li}^{+}\left({ }^{1} \mathrm{~S}^{\mathrm{e}}\right), \mathrm{Be}^{2+}\left({ }^{1} \mathrm{~S}^{\mathrm{e}}\right)$ and $\mathrm{B}^{3+}\left({ }^{1} \mathrm{~S}^{\mathrm{e}}\right)$ as targets. Comparing figures 1,2 , and 5 it is clear that the $\mathrm{CD}$ is much more robust a function to changes in the double ionization dynamics. The $\mathrm{CD}$ results shown in figure 5 resemble those of figure 1 . However, the predictions of figure 5 are expected to be more reliable than those of figures 1 and 2 since the approximations (3), (10) are more reasonable for stronger Coulomb fields of the nucleus.

To illustrate the behaviour of the $\mathrm{CD}$ in the case of strong interelectronic correlation 

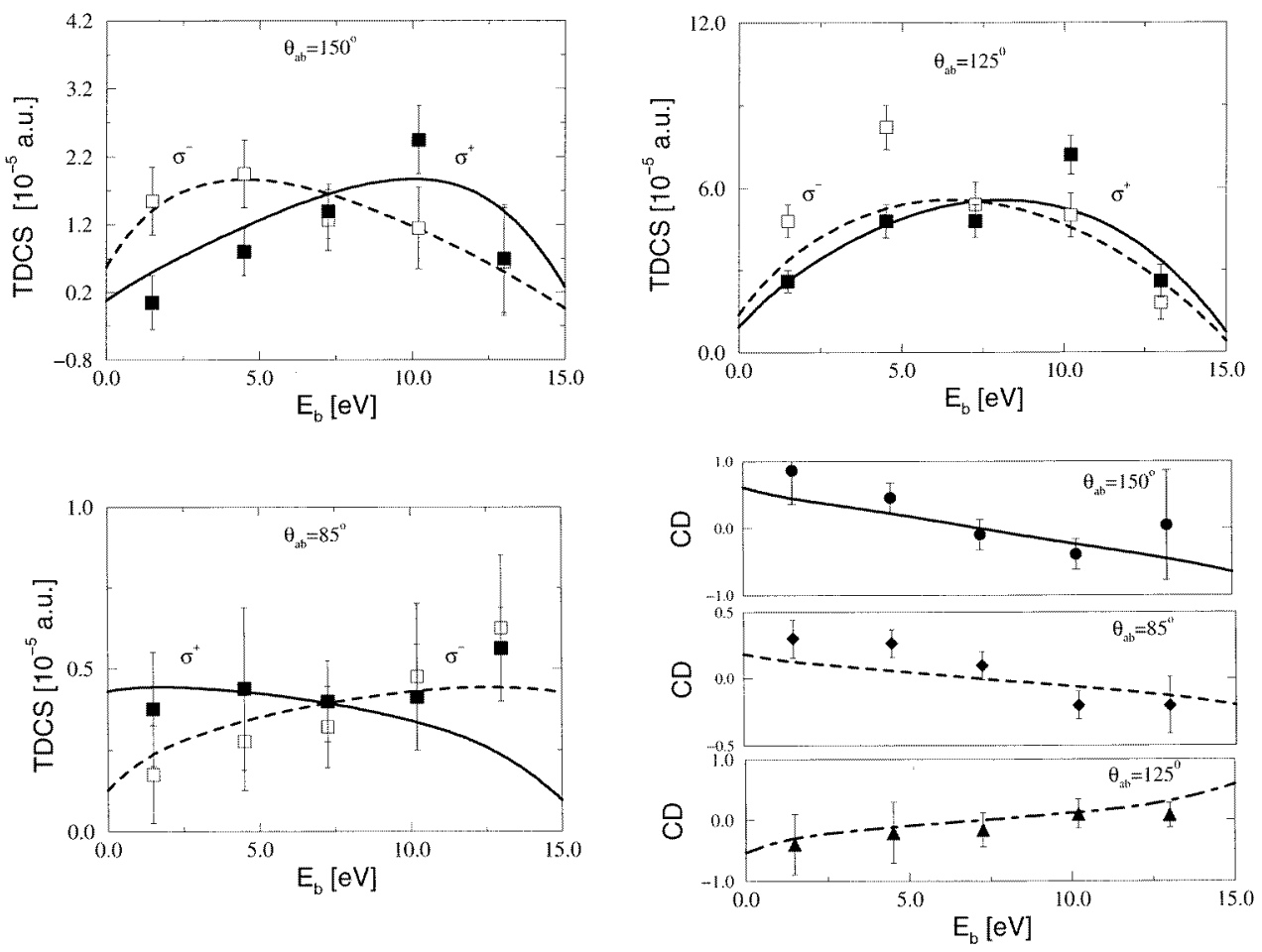

Figure 4. The same as in figure 1, however, the wavefunction (8) has been employed for the final state. The theoretical TDCS at $\theta_{a b}=150^{\circ}\left(85^{\circ}\right)$ have been scaled down by a factor of 2 (3).

we consider in figure 6 the case of $\mathrm{H}^{-}\left({ }^{1} \mathrm{~S}^{\mathrm{e}}\right)$. Equation (3) does not support any bound states for this system whereas equation (4) predicts a binding energy of -0.5239 au in good agreement with the experimental value of -0.5277 au. In the case of $\mathrm{H}^{-}\left({ }^{1} \mathrm{~S}^{\mathrm{e}}\right)$, the $\mathrm{CD}$ has a complicated structure and changes sign a few times for the same $\theta_{a b}$. This behaviour becomes more prevalent at even lower excess energies (Berakdar 1997) in which case the $\mathrm{CD}$ exhibits, basically, oscillatory behaviour.

The $Z$-dependence of the $\mathrm{CD}$ sign is also depicted in figure 6 . For $\mathrm{B}^{3+}$ the sign of the $\mathrm{CD}$ at $\theta_{a b}=125^{\circ}$ is inverted in comparison with that of $\mathrm{He}, \mathrm{Li}^{+}$and $\mathrm{Be}^{2+}$.

For $\mathrm{Li}^{+}$and $\mathrm{B}^{3+}$ the use of correlated wavefunctions (equations (4), (8)) results in substantially different predictions (figure 6) for the CD and the TDCS than those shown in figure 5. In particular, the magnitude of the TDCS is markedly enhanced due to the inclusion of electronic interactions ( $\mathrm{cf}$ figures 5 and 6). According to the correlated models (equations (4), (8)), the size of the TDCS varies much more rapidly with increasing $Z$ than in the case for electronically uncorrelated methods (see figure 5). In the absence of any experimental verification of the predictions depicted in figures 5 and 6 , it is not definite which of those predictions are more reliable, since it turned out (Berakdar 1997) that the differences in the TDCS, as observed in figures 5 and 6 , are mainly due to the use of the approximation (8) whose range of validity for higher $Z$ is unclear. 

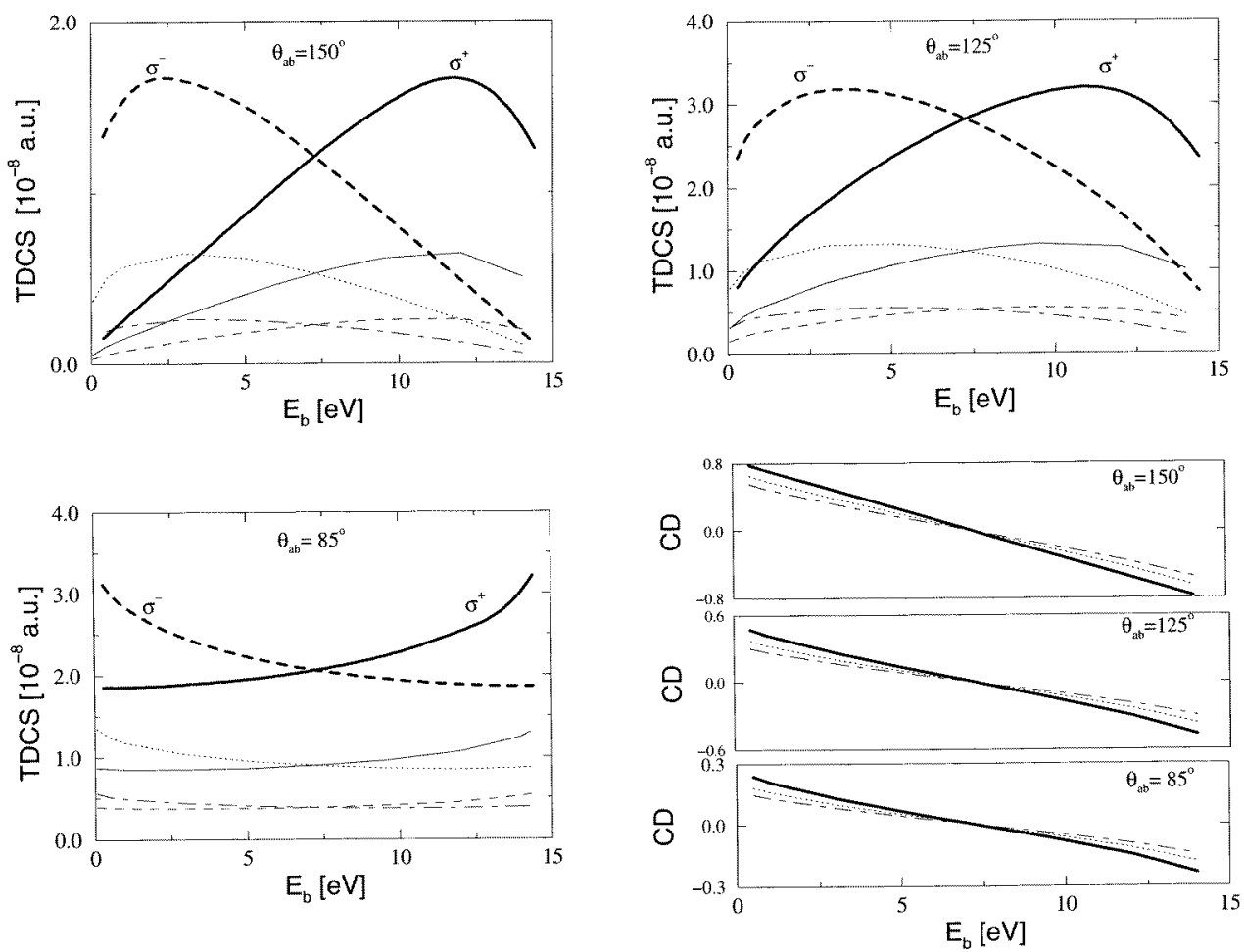

Figure 5. The one-photon double ionization of $\mathrm{Li}^{+}\left({ }^{1} \mathrm{~S}^{\mathrm{e}}\right), \mathrm{Be}^{2+}\left({ }^{1} \mathrm{~S}^{\mathrm{e}}\right), \mathrm{B}^{3+}\left({ }^{1} \mathrm{~S}^{\mathrm{e}}\right)$ in the same geometry and using the same dynamical models as described in figure 2. The excess energy is fixed at $14.52 \mathrm{eV}$, i.e. the photon energy is varied so as to compensate for the different doubleionization potential of the different targets. The figures depicted are: $\operatorname{TDCS}^{\mathrm{Li}^{+}}\left(\sigma^{+}\right)$: heavy full curve, $\operatorname{TDCS}^{\mathrm{Li}^{+}}\left(\sigma^{-}\right)$: heavy broken curve, $\operatorname{TDCS}^{\mathrm{Be}^{2+}}\left(\sigma^{+}\right)$: light full curve, $\operatorname{TDCS}^{\mathrm{Be}^{2+}}\left(\sigma^{-}\right)$: dotted curve, $\operatorname{TDCS}^{\mathrm{B}^{3+}}\left(\sigma^{+}\right)$: light broken curve, and $\operatorname{TDCS}^{\mathrm{B}^{3+}}\left(\sigma^{-}\right)$: chain curve. Also shown is the $\mathrm{CD}$ for the case of $\mathrm{Li}^{+}$(full curve), $\mathrm{Be}^{2+}$ (dotted curve), and $\mathrm{B}^{3+}$ (chain curve). The TDCS for $\mathrm{Be}^{2+}\left(\mathrm{B}^{3+}\right)$ has been multiplied by a factor of 2 (3).

\section{The photon-frequency dependence of the CD}

In a previous work (Berakdar et al 1993) it has been shown that the Wannier-PeterkopRau (Wannier 1953, Peterkop 1971, Rau 1971, 1984 Huetz et al 1991) theory yields vanishing $\mathrm{CD}$ close to the double-ionization threshold. In accordance with this prediction, dynamical calculations using the wavefunction (8) showed a decreasing CD at lower excess energies. At some specific intermediate photon frequencies, however, the $\mathrm{CD}$ changed sign. No explanation has yet been offered for this dependence of the $\mathrm{CD}$ on the photon frequency. How the photon-frequency dependence of the $\mathrm{CD}$ interplays with the geometrical arrangement of the experiment also remains an open question.

The photon-frequency dependence of $\Sigma$ and the CD, as described by equations (B4), (14), is more transparent in the parametrization $k_{a}=\sqrt{2 E} \sin \alpha, k_{b}=\sqrt{2 E} \cos \alpha$ ( $E$ is the excess energy). Equation (14) then reads, $\mathrm{CD}=Z \sqrt{2 E}(\cos \alpha-\sin \alpha)\left(\hat{\boldsymbol{k}}_{a} \times \hat{\boldsymbol{k}}_{b}\right) \cdot \hat{\boldsymbol{k}} / \mathcal{F}$, where

$$
\begin{gathered}
\mathcal{F}=\frac{2 E \cos ^{2} \alpha+Z_{s}^{2}}{2 E \sin ^{2} \alpha+Z_{s}^{2}}\left(2 E \sin ^{2} \alpha+Z^{2}\right)+\frac{2 E \sin ^{2} \alpha+Z_{s}^{2}}{2 E \cos ^{2} \alpha+Z_{s}^{2}}\left(2 E \cos ^{2} \alpha+Z^{2}\right) \\
+2 E\left(\sin 2 \alpha+Z^{2}\right) \cos \theta_{a b} .
\end{gathered}
$$



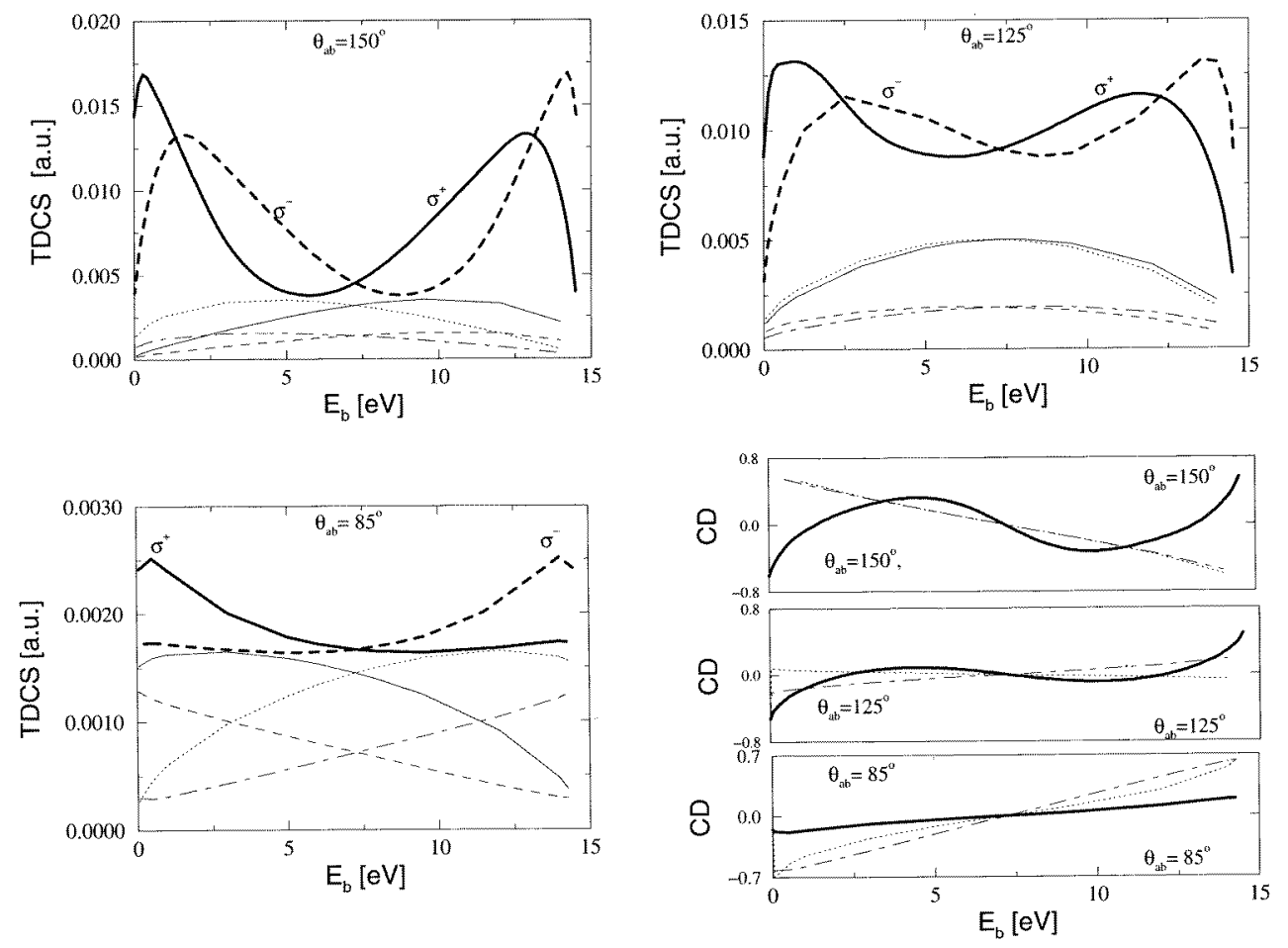

Figure 6. Same geometry and same excess energy as in figure 5. The wavefunctions (4) and (8) have been used for the initial- and final-state wavefunctions, respectively. Included are the TDCS results for $\mathrm{H}^{-}\left({ }^{1} \mathrm{~S}^{\mathrm{e}}\right)\left(\operatorname{TDCS}\left(\sigma^{+}\right)\right.$: heavy full curve, $\operatorname{TDCS}\left(\sigma^{-}\right)$: heavy broken curve $), \mathrm{Li}^{+}\left({ }^{1} \mathrm{~S}^{\mathrm{e}}\right)$ (TDCS $\left(\sigma^{+}\right)$: light full curve, $\operatorname{TDCS}\left(\sigma^{-}\right)$: dotted curve), and $\mathrm{B}^{3+}\left(\operatorname{TDCS}\left(\sigma^{+}\right)\right.$: broken light curve, TDCS $\left(\sigma^{-}\right)$: chain curve). The TDCS results for $\mathrm{Li}^{+}\left(\mathrm{B}^{3+}\right)$ have been multiplied by a factor of $3000\left(10^{5}\right)$. The corresponding $\mathrm{CD}\left(\mathrm{H}^{-}\left({ }^{1} \mathrm{~S}^{\mathrm{e}}\right)\right.$ (heavy full curve), $\mathrm{Li}^{+}\left({ }^{1} \mathrm{~S}^{\mathrm{e}}\right)$ (dotted curve), and $\mathrm{B}^{3+}$ (chain curve)) are also shown.

Since $\mathcal{F}=F / \Sigma$ and the function $F(E)$, as given by equation (A12), is positive definite it follows that $\mathcal{F}(E)$ is positive definite as well. At the threshold $(E \rightarrow 0)$ the $\mathrm{CD}$ decreases as $\sqrt{E}$. For high photon frequency $(E \rightarrow \infty)$ the $\mathrm{CD}$ is proportional to $1 / \sqrt{E}$. Except for these two limits the CD possesses no additional zero points as a function of $E$ (this is due to $\mathcal{F}(E)>0, \forall E$ and $\mathrm{CD} \propto \sqrt{E} / \mathcal{F})$. These conclusions are applicable when employing the approximate wavefunctions (9), (10) for the final state. When using the wavefunction given by equation (8) additional zero points of the $\mathrm{CD}$ as a function of $E$ arise and must, hence, be assigned to the electron-electron interaction.

\section{Conclusions}

In this work we considered the $\mathrm{CD}$ and the fully differential cross section for the onephoton two-electron emission from $\mathrm{H}^{-}\left({ }^{1} \mathrm{~S}^{\mathrm{e}}\right), \mathrm{He}\left({ }^{1} \mathrm{~S}^{\mathrm{e}}\right), \mathrm{Li}^{+}\left({ }^{1} \mathrm{~S}^{\mathrm{e}}\right), \mathrm{Be}^{2+}\left({ }^{1} \mathrm{~S}^{\mathrm{e}}\right)$, and $\mathrm{B}^{3+}\left({ }^{1} \mathrm{~S}^{\mathrm{e}}\right)$. Analytical formulae for the dichroism and the cross sections have been derived and interpreted within reasonably simple models. Contrasting the predictions of the analytical formulae with elaborate numerical calculations, it has been possible to deduce that the interelectronic interaction leads to vanishing $C D$ at certain 'non-geometrical' points as well as at certain photon frequencies. The results of this study indicate that the CD is element 
sensitive and does not follow an obvious trend with increasing $Z$.

It remains an open question for future theoretical and experimental work on the $\mathrm{CD}$ why electron-electron correlation is preferentially pronounced at the 'non-geometrical' region where the $\mathrm{CD}$ vanishes.

\section{Acknowledgments}

I would like to thank Lorenzo Avaldi, Uwe Becker, Volker Mergel, Reinhardt Dörner, Horst Schmidt-Böcking, Jens Viefhaus and Hubert Klar for fruitful discussions.

\section{Appendix A. The analytical form of the CD}

In this appendix we derive analytical expressions for the TDCS and the CD using the wavefunction (3), (9), (10). The integrals involved in calculating the TDCS can be reduced to Fourier transforms of the type

$$
\begin{aligned}
\mathcal{I}=\int \mathrm{d}^{3} r \hat{\boldsymbol{\epsilon}} \cdot & \boldsymbol{r} \exp (-\beta r) \exp (\mathrm{i} \boldsymbol{p} \cdot \boldsymbol{r})_{1} F_{1}(-\mathrm{i} \alpha, 1, \mathrm{i}[k r+\boldsymbol{k} \cdot \boldsymbol{r}])=\mathrm{i} \frac{\partial^{2}}{\partial \beta_{d} \partial \lambda} \int \mathrm{d}^{3} r \\
& \times\left.\frac{\exp \left(-\beta_{d} r\right)}{r} \exp (\mathrm{i} \boldsymbol{p} \cdot \boldsymbol{r}+\mathrm{i} \lambda \hat{\boldsymbol{\epsilon}} \cdot \boldsymbol{r})_{1} F_{1}(-\mathrm{i} \alpha, 1, \mathrm{i}[k r+\boldsymbol{k} \cdot \boldsymbol{r}])\right|_{\lambda=0, \beta_{d}=\beta} \\
= & \left.\mathrm{i} \frac{\partial^{2}}{\partial \beta_{d} \partial \lambda} \frac{4 \pi}{\beta_{d}^{2}+P^{2}}\left[\frac{(\boldsymbol{P}+\boldsymbol{k})^{2}-\left(k+\mathrm{i} \beta_{d}\right)^{2}}{\beta_{d}^{2}+P^{2}}\right]^{\mathrm{i} \alpha}\right|_{\lambda=0, \beta_{d}=\beta},
\end{aligned}
$$

where $\boldsymbol{P}:=\boldsymbol{p}+\lambda \hat{\boldsymbol{\epsilon}}$. Employing the wavefunction (3), (10) for the initial and the final state and making use of equation (A1), the TDCS (equation (2)) can be written in the form

$$
\operatorname{TDCS}=C_{a}\left|J_{b} I_{a}+J_{a} I_{b}\right|^{2},
$$

where

$$
C_{a}=128 k_{a} k_{b} \omega \alpha_{c}\left|N_{s} N_{a} N_{b} N_{a b}\right|^{2} .
$$

After some algebraic manipulation, the functions $I_{j}, J_{j}, j=a, b$ in equation (A2) can be expressed as

$$
I_{j}=-\mathrm{i}\left(1+\mathrm{i} \beta_{j}\right) \boldsymbol{B}_{j} \cdot \hat{\boldsymbol{\epsilon}},
$$

and

$$
J_{j}=f_{j}\left[\frac{Z_{s}+\beta_{j} k_{j}}{\left(k_{j}^{2}+Z_{s}^{2}\right)^{2}}\right] .
$$

In equation (A5) the real scalars $f_{j}$ are given by

$$
f_{j}:=\exp \left[2 \beta_{j} \arctan \left(\frac{k_{j}}{Z_{s}}\right)\right],
$$

whereas in equation (A4) the real vectors $\boldsymbol{B}_{j}$ read

$$
\boldsymbol{B}_{j}=2 f_{j}\left[\frac{2 Z_{s}-Z}{\left(k_{j}^{2}+Z_{s}^{2}\right)^{3}}\right] \boldsymbol{k}_{j} .
$$


We further define the real vectors $\boldsymbol{A}_{a}=\boldsymbol{B}_{a} J_{b}, \boldsymbol{A}_{b}=\boldsymbol{B}_{b} J_{a}$ and the un-normalized dichroism as $\Delta=\operatorname{TDCS}\left(\sigma^{+}\right)-\operatorname{TDCS}\left(\sigma^{-}\right)$. From equation (A2) we derive an expression for $\Delta$ :

$$
\begin{aligned}
\Delta=C_{a}[(1+ & \left.\left.\mathrm{i} \beta_{a}\right) \boldsymbol{A}_{a} \cdot \hat{\boldsymbol{\epsilon}}+\left(1+\mathrm{i} \beta_{b}\right) \boldsymbol{A}_{b} \cdot \hat{\boldsymbol{\epsilon}}\right]\left[\left(1-\mathrm{i} \beta_{a}\right) \boldsymbol{A}_{a} \cdot \hat{\boldsymbol{\epsilon}}^{*}+\left(1-\mathrm{i} \beta_{b}\right) \boldsymbol{A}_{b} \cdot \hat{\boldsymbol{\epsilon}}^{*}\right] \\
& -C_{a}\left[\left(1+\mathrm{i} \beta_{a}\right) \boldsymbol{A}_{a} \cdot \hat{\boldsymbol{\epsilon}}^{*}+\left(1+\mathrm{i} \beta_{b}\right) \boldsymbol{A}_{b} \cdot \hat{\boldsymbol{\epsilon}}^{*}\right] \\
& \times\left[\left(1-\mathrm{i} \beta_{a}\right) \boldsymbol{A}_{a} \cdot \hat{\boldsymbol{\epsilon}}+\left(1-\mathrm{i} \beta_{b}\right) \boldsymbol{A}_{b} \cdot \hat{\boldsymbol{\epsilon}}\right] .
\end{aligned}
$$

The latter equation can be simplified to

$$
\Delta=-2 \mathrm{i} C_{a}\left(\beta_{b}-\beta_{a}\right)\left[\left(\boldsymbol{A}_{a} \times \hat{\boldsymbol{\epsilon}}\right)\left(\boldsymbol{A}_{b} \times \hat{\boldsymbol{\epsilon}}^{*}\right)-\left(\boldsymbol{A}_{a} \times \hat{\boldsymbol{\epsilon}}^{*}\right)\left(\boldsymbol{A}_{b} \times \hat{\boldsymbol{\epsilon}}\right)\right] .
$$

Making use of the recoupling formula

$(\boldsymbol{a} \cdot \boldsymbol{b})(\boldsymbol{c} \cdot \boldsymbol{d})=\frac{1}{3}(\boldsymbol{a} \cdot \boldsymbol{c})(\boldsymbol{b} \cdot \boldsymbol{d})+\frac{1}{2}(\boldsymbol{a} \times \boldsymbol{c})(\boldsymbol{b} \times \boldsymbol{d})+T_{2}(\boldsymbol{a}, \boldsymbol{c}) \cdot T_{2}(\boldsymbol{b}, \boldsymbol{d})$,

where $T_{2}(\boldsymbol{i}, \boldsymbol{j})$ is a tensor of rank 2, equation (A9) takes on the form

$$
\Delta=-Z F\left(k_{a}-k_{b}\right)\left(\hat{\boldsymbol{k}}_{a} \times \hat{\boldsymbol{k}}_{b}\right) \cdot \hat{\boldsymbol{k}} .
$$

The function $F$ is then given by

$$
F=2 C_{a}\left(Z_{s}-Z\right)^{2}\left(2 Z_{s}-Z\right)^{2}\left(2 f_{a} f_{b}\right)^{2}\left(k_{a}^{2}+Z_{s}^{2}\right)^{-5}\left(k_{b}^{2}+Z_{s}^{2}\right)^{-5} .
$$

For the present study it is important to note that $F$ (equation (A12)) is angular independent and positive definite for all $k_{a}, k_{b}$.

\section{Appendix B. The cross sections}

Equation (A11) is the expression for the un-normalized dichroism. To emphasize the independence of the $\Delta$ and $\operatorname{TDCS}\left(\sigma^{ \pm}\right)$we define a normalized $\mathrm{CD}$ as

$$
\mathrm{CD}=\frac{\Delta}{\Sigma}
$$

where

$$
\Sigma:=\operatorname{TDCS}\left(\sigma^{+}\right)+\operatorname{TDCS}\left(\sigma^{-}\right) .
$$

From equation (A2) and after lengthy, but straightforward algebraic manipulation it follows:

$$
\begin{gathered}
\Sigma=2 C_{a}\left\{\left(1+\beta_{a}^{2}\right)\left|\boldsymbol{A}_{a} \cdot \hat{\boldsymbol{\epsilon}}\right|^{2}+\left(1+\beta_{b}^{2}\right)\left|\boldsymbol{A}_{b} \cdot \hat{\boldsymbol{\epsilon}}\right|^{2}+\left(1+\beta_{a} \beta_{b}\right)\left[\left(\boldsymbol{A}_{a} \cdot \hat{\boldsymbol{\epsilon}}\right)\left(\boldsymbol{A}_{b} \cdot \hat{\boldsymbol{\epsilon}}^{*}\right)\right.\right. \\
\left.\left.+\left(\boldsymbol{A}_{b} \cdot \hat{\boldsymbol{\epsilon}}\right)\left(\boldsymbol{A}_{a} \cdot \hat{\boldsymbol{\epsilon}}^{*}\right)\right]\right\} .
\end{gathered}
$$

Making use of equations (A4), (A5), (A7), equation (B3) can be reduced to

$$
\begin{gathered}
\Sigma=2 C_{a}\left(2 f_{a} f_{b}\right)^{2} \frac{\left(Z_{s}-Z\right)^{2}\left(2 Z_{s}-Z\right)^{2}}{\left(k_{a}^{2}+Z_{s}^{2}\right)^{4}\left(k_{b}^{2}+Z_{s}^{2}\right)^{4}}\left\{\frac{k_{a}^{2}+Z^{2}}{\left(k_{a}^{2}+Z_{s}^{2}\right)^{2}}\left|\hat{\boldsymbol{k}}_{a} \cdot \hat{\boldsymbol{\epsilon}}\right|^{2}+\frac{k_{b}^{2}+Z^{2}}{\left(k_{b}^{2}+Z_{s}^{2}\right)^{2}}\left|\hat{\boldsymbol{k}}_{b} \cdot \hat{\boldsymbol{\epsilon}}\right|^{2}\right. \\
\left.+2 \frac{k_{a} k_{b}+Z^{2}}{\left(k_{a}^{2}+Z_{s}^{2}\right)\left(k_{b}^{2}+Z_{s}^{2}\right)} \operatorname{Re}\left[\left(\hat{\boldsymbol{k}}_{a} \cdot \hat{\boldsymbol{\epsilon}}\right)\left(\hat{\boldsymbol{k}}_{b} \cdot \hat{\boldsymbol{\epsilon}}^{*}\right)\right]\right\} .
\end{gathered}
$$

Now combining equations (B4), (A11) we end up with the final result for the CD

$$
\mathrm{CD}:=-\frac{Z}{\mathcal{F}}\left(k_{a}-k_{b}\right)\left(\hat{\boldsymbol{k}}_{a} \times \hat{\boldsymbol{k}}_{b}\right) \cdot \hat{\boldsymbol{k}},
$$

where $\mathcal{F}$ is positive definite in the six-dimensional $\boldsymbol{k}_{a} \otimes \boldsymbol{k}_{b}$ space and has the form

$$
\begin{gathered}
\mathcal{F}=\frac{k_{b}^{2}+Z_{s}^{2}}{k_{a}^{2}+Z_{s}^{2}}\left(k_{a}^{2}+Z^{2}\right)\left|\hat{\boldsymbol{k}}_{a} \cdot \hat{\boldsymbol{\epsilon}}\right|^{2}+\frac{k_{a}^{2}+Z_{s}^{2}}{k_{b}^{2}+Z_{s}^{2}}\left(k_{b}^{2}+Z^{2}\right)\left|\hat{\boldsymbol{k}}_{b} \cdot \hat{\boldsymbol{\epsilon}}\right|^{2} \\
+2\left(k_{a} k_{b}+Z^{2}\right) \operatorname{Re}\left[\left(\hat{\boldsymbol{k}}_{a} \cdot \hat{\boldsymbol{\epsilon}}\right)\left(\hat{\boldsymbol{k}}_{b} \cdot \hat{\boldsymbol{\epsilon}}^{*}\right)\right] .
\end{gathered}
$$




\section{References}

Berakdar J 1996 Phys. Rev. A 532314

-1997 unpublished

1998 Phys. Rev. B submitted

Berakdar J and Klar H 1992 Phys. Rev. Lett. 691175

Berakdar J, Klar H, Huetz A and Selles P 1993 J. Phys. B: At. Mol. Opt. Phys. 261463

Bethe H A and Salpeter E E 1957 Quantum Mechanics of One- and Two-Electron Atoms (Berlin: Springer)

Brauner M, Briggs J S and Klar H 1989 J. Phys. B: At. Mol. Opt. Phys. 222265

Herrmann R, Samarin S, Schwabe H and Kirschner J 1998 Phys. Rev. Lett. submitted

Huetz A, Selles P, Waymel D and Mazeau J 1991 J. Phys. B: At. Mol. Opt. Phys. 241917

Jackson J D 1967 Classical Electrodynamics 6th edn (New York: Wiley)

Kabachnik N M, and Schmidt V 1995 J. Phys. B: At. Mol. Opt. Phys. 28233

Manakov N L, Marmo S I and Meremianin A V 1996 J. Phys. B: At. Mol. Opt. Phys. 292711

Maulbetsch F 1995 PhD Thesis Freiburg University and references therein

Maulbetsch F, Pont M, Briggs J S and Shakeshaft R 1995 J. Phys. B: At. Mol. Opt. Phys. 28 L341

Mergel V et al 1998 Phys. Rev. Lett. 805301

Peterkop R 1971 J. Phys. B: At. Mol. Phys. 4513

Rau A R P 1971 Phys. Rev. A 4207

1984 Phys. Rep. 110369

Siebbeles L D A, Marshall D P and Le Sech C 1993 J. Phys. B: At. Mol. Opt. Phys. 26 L321

Soejima K, Shimbo M, Danjo A, Okuno K, Shigemasa E and Yagishita A 1996 J. Phys. B: At. Mol. Opt. Phys. 29 L367

Venus D, Kuch W, Lin T-M, Schneider C M, Ebert H and Kirschner J 1997 Phys. Rev. B 552594

Viefhaus J et al 1996 Phys. Rev. Lett. 773975

Wannier G 1953 Phys. Rev. 90817 